\title{
The prediction of the wild-type telomerase RNA pseudoknot structure and the pivotal role of the bulge in its formation
}

\author{
Yaroslava G. Yingling, Bruce A. Shapiro* \\ Center for Cancer Research Nanobiology Program, National Cancer Institute, NCI-Frederick, National Institutes of Health, \\ Building 469, Room 150, Frederick, MD 21702, United States \\ Received 14 November 2005; received in revised form 6 January 2006; accepted 8 January 2006 \\ Available online 14 February 2006
}

\begin{abstract}
In this study, the three-dimensional structure of the wild-type human telomerase RNA pseudoknot was predicted via molecular modeling. The wild-type pseudoknot structure is then compared to the recent NMR solution structure of the telomerase pseudoknot, which does not contain the U177 bulge. The removal of the bulge from the pseudoknot structure results in higher stability and significant reduction of activity of telomerase. We show that the effect of the bulge on the structure results in a significant transformation of the pseudoknot junction region where the starting base pairs are disrupted and unique triple base pairs are formed. We found that the formation of the junction region is greatly influenced by interactions of the U177 bulge with loop residues and rotation of residue A174. Moreover, this is the first study to our knowledge where a structure as complex as the pseudoknot has been solved by purely theoretical methods.
\end{abstract}

Published by Elsevier Inc.

Keywords: Telomerase; RNA; Molecular dynamics; Theoretical prediction; Pseudoknot

\section{Introduction}

Telomeres are highly organized nucleoprotein complexes which are located at the ends of eukaryotic chromosomes. Telomeres are not stable due to the loss of telomeric DNA at each cell division [1], until a critically short length occurs causing cell cycle arrest or apoptosis. Cell divisions with short telomeres may cause end-to-end fusions, karyotypic changes, cell death, and genomic instability. The maintenance of telomeres is achieved by reactivation of the enzyme called telomerase, which balances telomere shortening with telomere elongation by adding telomeric DNA repeat sequences to the ends of chromosomes. Telomerase is active in about $85 \%$ of all human tumors, which makes telomerase an attractive target for cancer therapy, diagnosis, and prognosis [2,3]. Telomerase is also functional in other proliferative tissues, as in stem cells, germ line cells, inflammatory cells, and cells in other periodically or continuously renewing tissues.

Human telomerase consists of a 451 nucleotide RNA (hTR), a telomerase reverse transcriptase (hTERT) protein, and a

\footnotetext{
* Corresponding author. Tel.: +1 301846 5536; fax: +1 3018465598 .

E-mail address: bshapiro@ncifcrf.gov (B.A. Shapiro).
}

variety of other proteins. The hTR contains the $5^{\prime}$ pseudoknot (core) domain which provides the template and enhances repeat amplification processivity [4], and the CR4/CR5 domain [5] which supports the catalytic activity by enhancing nucleotide addition processivity. Both domains are essential for catalytic activity and hTERT binding. In addition, the hTR contains the $3^{\prime} \mathrm{H} / \mathrm{ACA}$ and CR7 domains which are involved in localization, accumulation, and $3^{\prime}$ end processing. Mutations in hTR have been linked to autosomal dominant dyskeratosis congenita (DKC) and aplastic anemia, both syndromes are characterized by hematopoietic function losses [6,7].

The structure of the pseudoknot core domain includes the single stranded template sequence, paired template boundary region $(\mathrm{P} 1 b)$, and an extended helical region $(\mathrm{P} 2)$ that folds into a pseudoknot (P3) structure [8]. The template and the pseudoknot are conserved in other telomerase RNAs [9]. Synthesis of telomere repeats takes place on the template and the pseudoknot domain is important for regulating the dynamic structure of the telomerase holoenzyme [10-12].

The focus of the present study is the pseudoknot structure (P3) located in hTR [8], which is of critical importance for stability of the ribonucleoprotein complex and telomerase activity $[9,13]$. Chemical and enzymatic probing analysis [14] and biophysical studies $[10,15]$ suggested that the pseudoknot 
in solution can exist in two alternative stable states. The first state is a hairpin pentaloop domain alone which includes paired sequences including $\mathrm{P} 2 \mathrm{~b}$. The second state is the pseudoknot which is formed by base pairing of the hairpin loop with the P3 domain creating a 9 bp helix with a single bulged $U$ residue in the upper strand. The primary sequence of both strands in the P3 region is highly conserved in vertebrate evolution and exhibits complementarity (conserved residues in P3 are shaded in grey in Fig. 1) [8]. Mutations that are proposed to disrupt base pairing in the $\mathrm{P} 3$ region reduce or abolish telomerase activity, whereas compensatory mutations generally restore it $[13,16]$. Consequently, hTR function is dependent on P3 base pairing with the hairpin loop, i.e. pseudoknot formation. Conversely, in vivo and in vitro chemical and enzymatic accessibility mapping have failed to confirm a stable P3 helix in hTR [14]. Nevertheless, experimental thermodynamics, phylogenetic analysis, and biochemical analyses of telomerase RNA agree that the pseudoknot is formed only temporarily in the telomerase and that the dynamic conformational switch between these two states is critical for telomerase functioning $[11,15,16]$. Then again, recent mutational analyses argue against the previously proposed molecular-switch model of the telomerase pseudoknot function and supports a static pseudoknot structure [17].

NMR spectroscopy and X-ray crystallography techniques are routinely used to provide the three-dimensional solution structures of biomolecules. However, flexible and dynamic molecules are less accessible to structure determination by these methods. The dynamic character and the conformation switch of the wild-type pseudoknot of telomerase RNA make it challenging for NMR or X-ray techniques to determine the solution structure. Several experimental studies used the pseudoknot structure with the bulge base U177 removed from the $\mathrm{P} 3$ region. The deletion of the bulge shifts the pseudoknothairpin equilibrium toward the pseudoknot and significantly reduces telomerase activity [11,18], therefore, making determination of the pseudoknot structure possible. Recent studies revealed the solution structure of the pseudoknot without the bulge and showed the formation of an extended triple helix surrounding the helical junction [18]. This study concludes that the high sequence conservation in the pseudoknot is crucial for formation of specific tertiary interactions around the junction that is essential for telomerase activity and stability. The NMR pseudoknot solution structure without the U177 bulge will be called the " $\Delta \mathrm{U} 177$ pseudoknot" throughout the text.

The presence and the position of this bulge in the P3 helix are conserved in vertebrates, though the base type varies [8]. Furthermore, the presence of the bulge is of crucial significance for the activity and functionality of the telomerase $[11,18]$. Overall, bulges are important for the recognition and binding of RNA by proteins $[19,20]$ and for initiating RNA tertiary folding [21]. For example, comparative analysis of a small subunit of ribosomal RNA suggests that a bulge pentaloop initiates pseudoknot formation with a terminal loop [21]. Another experimental study showed that the U-rich bulge in the TAR RNA structure is responsible for HIV-1 tat protein binding and HIV transcription [19]. Moreover, bulges can distort the usual A-type conformation of RNA helices by bending the helical axis [22,23], change the major or minor groove dimensions and permit atypical tertiary contacts $[20,24]$. For example, transient

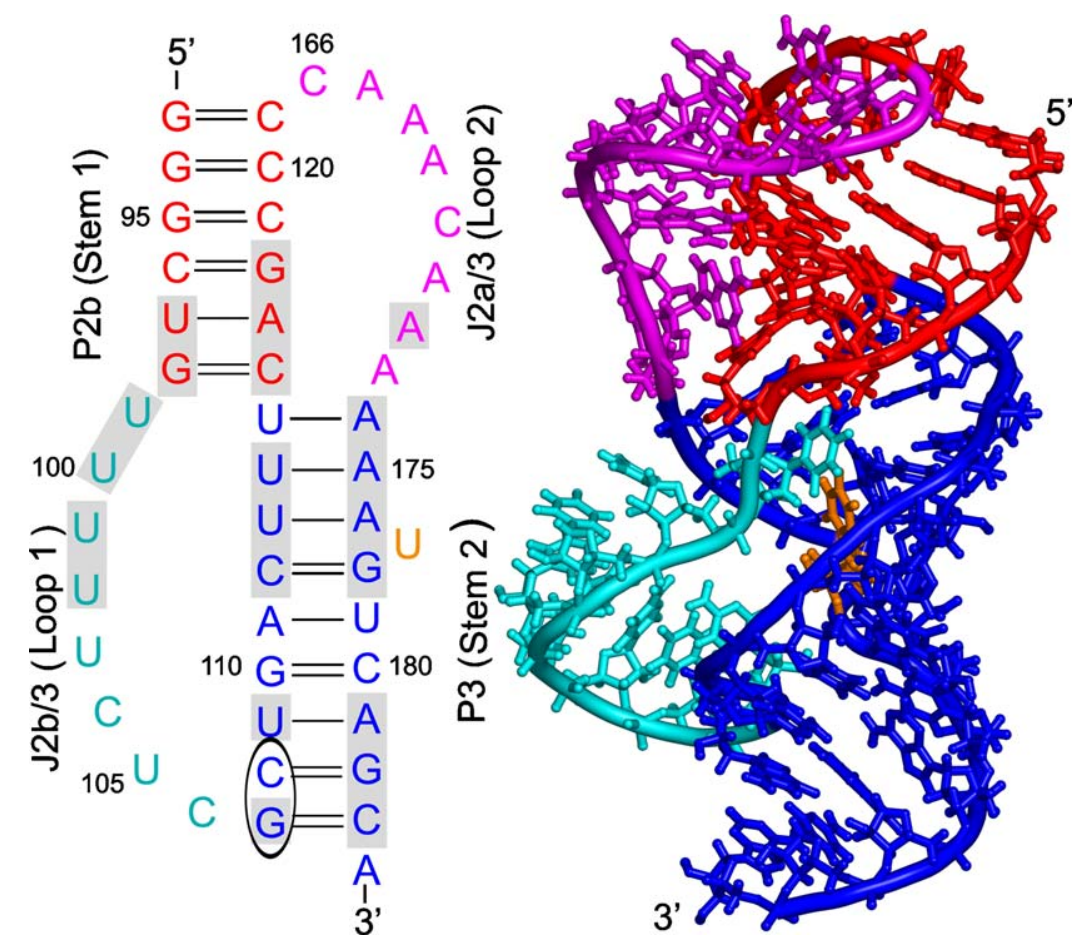

Fig. 1. The model wild-type pseudoknot secondary structure and the snapshot of the three-dimensional starting structure. Bases are numbered according to the full structure [8]. The 100\% conserved nucleotides are shaded in gray. The coloring of the nucleotides is based on their association with Stem 1 (red), Loop 1 (cyan), Loop 2 (magenta), and Stem 2 (red). Circled residues participate in DKC mutations. 
electric birefringence analysis shows that a single $U$ bulge in a duplex RNA increases the helical bend angle by $7^{\circ}$ (in the presence of $\mathrm{Mg}^{2+}$ ) or $10^{\circ}$ (in the absence of $\mathrm{Mg}^{2+}$ ) [23]. We can then expect that the solution structure of the wild-type telomerase RNA pseudoknot with the U bulge in the P3 helix would be different than the structure of the pseudoknot without the bulge.

We employed molecular dynamics (MD) simulations to predict the 3D solution structure of the wild-type telomerase RNA pseudoknot. MD is a technique in which the time evolution of the molecular system is followed from numerical integration of the equations of motion. MD makes possible the dynamic characterization and an exploration of the conformational energy landscape of biomolecules and their surroundings. Moreover, MD simulations have been successfully used to characterize a wide range of nucleic acid structures as outlined in recent reviews [25-30]. However, the reliability of MD depends on accurate and representative force fields for both nucleic acids and solvent. Accurate simulations with explicit solvent are computationally expensive. Simulations have to be long enough for the conformational transitions to occur in a biomolecular system. Up to now the longest explicit solvent simulations were around a $20 \mathrm{~ns}$ timescale $[28,50]$. MD simulations of biomolecules in a liquid environment can be significantly accelerated by using approximations of the electrostatic effects of the solvent. In these approximations the solvent is typically treated by the continuum dielectric methods and only the intrasolute electrostatics need to be evaluated, which consequently reduce the number of interactions with respect to explicit solvent methods [31]. These methods have proven to be reliable and able to provide crucial information for various biomolecules [26,32-34]. The generalized Born (GB) theory [35-37] is one of the most successful approximations of the Poisson equation for continuum electrostatic solvation energy and describes the electrostatic energy of two or more atomic charges in a cavity of arbitrary shape. GB involves accurate evaluation of the average spherical distances of each atom to the solvent boundary. Consequently, the GB energy expression, involving summation of self and pair wise interactions of atomic charges, is an analytical function of atomic positions and is very good at reproducing the Poisson energy with much smaller computational cost [38].

Implicit solvent simulations can also improve conformational searches and have already successfully predicted conformational preferences of small experimentally known nucleic acid structures [26]. For example, implicit simulations of the relative stability of various forms of RNA hairpin loop structures predicted the same low free energy conformations as experimentally observed [39]. Overall, even though implicit MD simulations are less accurate than simulations with explicit solvent, they permit not only much longer simulations and larger molecules but also provide a variety of sampled conformations. Considerable improvements in the force field have also been achieved making simulations more reliable and accurate. Moreover, MD simulations are often used for NMR structure refinement and simulated annealing [40], where the experimentally determined distance and dihedral restraints are added to the normal interaction potentials, and MD simulations are used to solve the structure of the biomolecules. Overall, MD techniques could be used to improve structure prediction and also assist in model building of biomolecules.

In this paper we report the solution structure of the telomerase wild-type pseudoknot that results from $56 \mathrm{~ns}$ of atomistic molecular dynamics simulations in implicit solvent. The reduced version of the pseudoknot structure used in this study has 48 residues and requires considerable time for equilibration. The use of implicit solvent allows us to test other cases of pseudoknot formation, like DKC-mutations. However, to reduce the potential artifacts of implicit solvation, the low energy wild-type pseudoknot structure was refined with 4 ns explicit solvent simulations. The starting structure was built from scratch and retained all proposed standard base pairs (Fig. 1). After $56 \mathrm{~ns}$ of molecular dynamics simulations the pseudoknot structure consists of two triple helices connected by a junction region which is also stabilized by triple base interactions. The final structure of the wild-type pseudoknot exhibits high stability for $40 \mathrm{~ns}$ which allows us to assume that the structure is located in an energy minimum. Due to time-limitations of MD we cannot guarantee that the determined structure is in its global energy minimum. However, the foremost advantage of our methodology is not only to predict the tertiary structure but also the ability to observe the dynamical interplay of base interactions during structure formation and stabilization. This is the first study to our knowledge where a structure as complex as the pseudoknot has been attempted to be solved by purely theoretical methods. We hope that this study not only provides insights into the structure and the dynamic characteristics of the telomerase RNA pseudoknot, but also depicts means by which one can generate the solution of a complete structure that cannot be determined experimentally due to its dynamic nature or size/space limitations.

\section{Methods}

\subsection{RNA2D3D}

The starting three-dimensional coordinates are generated from the secondary structure (Fig. 1) using the program RNA2D3D [41]. The initial standard interactions in the wildtype pseudoknot were exactly the interactions depicted in the secondary structure with minimal tertiary interactions involved.

RNA2D3D is designed to facilitate the generation, viewing, and comparison of 3D RNA molecules. It is based on the observation that the atomic coordinates of a nucleotide can be generated from a reference triad, i.e. three of its atoms. Then any stem can be generated from the reference triad of any of its nucleotides by using helical coordinates. The unpaired nucleotides, bulges, hairpin loops, branching loops, and other non-helical motifs are generated by using the coordinates of their reference triad relative to the $5^{\prime}$ neighboring nucleotide.

In this program, an RNA's secondary structure is initially used to generate a planar template of a backbone from base 
pairing information. This planar template is scaled to molecular dimensions and contains the absolute atomic coordinates of every nucleotide. The absolute atomic coordinates provide the information for determining relative coordinates for reference triads of nucleotides in loops and stems. The planar template is then recursively converted to its $3 \mathrm{D}$ form using a special $3 \mathrm{D}$ embedding procedure. This procedure incorporates the atomic models of nucleotides which are initially equally spaced along the fixed backbone. A stem is generated by using predetermined A-type helical coordinates. The atomic coordinates of each nucleotide, base pair, and A-type helical parameters are taken from the Biosym $(B)$ database. When a stem of a planar template is converted to its $3 \mathrm{D}$ form, the coordinates of the stem's $5^{\prime}$ loop-bounding nucleotide is used as a reference triad for building non-helical motifs, and the coordinates of the $3^{\prime}$ end of the non-helical motif is used to build the next motif. As a result, a first-order approximation of the actual $3 \mathrm{D}$ molecule is established. Structure refinement involves molecular modeling (Amber minimization) or interactive editing. The interactive editing involves a rotation and translation of a segment (a group of nucleotides) or a group of segments as a rigid body. This refinement is used for the removal of structural clashes, enforcing tertiary interactions, and modification of mutual stacking. For example, the pseudoknot stems can be moved relative to each other to modify their mutual stacking or the nucleotides in the loop can be rotated to remove tertiary interactions. The result of the recursive 3D structure generation and preliminary refinement is a first order approximation to the actual 3D model which can be further refined with molecular dynamics simulations.

\subsection{Molecular dynamics simulations}

All simulations were performed using the ff 99 Cornell force field for RNA [42], which has proven to be a reliable and refined force field for nucleic acids, and the molecular dynamics software Amber 7.0 [43] and Amber 8.0 [44].

\subsubsection{Implicit solvent}

Molecular dynamics simulations at $300 \mathrm{~K}$ constant temperature using the GB implicit solvent approach as implemented in the SANDER module of Amber 7.0 [43] were performed for all structures. Each starting structure was subjected to minimization (10,000 steps), followed by slow $20 \mathrm{kcal} / \mathrm{mol}$ constrained heating to $300 \mathrm{~K}$ over $200 \mathrm{ps}$ time, and several consecutive MD equilibrations with declining constraints from 2 to $0.1 \mathrm{kcal} / \mathrm{mol}$ over a total $500 \mathrm{ps}$ time period. The temperature was maintained at $300 \mathrm{~K}$ using a Berendsen thermostat [45]. The monovalent salt concentration was set to $0.5 \mathrm{~mol} / \mathrm{L}$. The production simulations were performed for $56 \mathrm{~ns}$ using $1 \mathrm{fs}$ time step.

\subsubsection{Explicit solvent}

The final low energy wild-type pseudoknot structure was refined with explicit solvent MD simulations for $4 \mathrm{~ns}$ using Amber 8.0 [44]. The structure was first neutralized with 47 $\mathrm{Na}^{+}$ions. A water box containing 34,368 molecules and an additional $30 \mathrm{Na}^{+}$and $30 \mathrm{Cl}^{-}$ions were added to represent a $0.1 \mathrm{M}$ solution. The electrostatic interactions were calculated by particle mesh ewald summation (PME) [46] and the nonbonded interactions were truncated at $9 \AA$. The system was minimized constraining the solute then solvent, then heated to $300 \mathrm{~K}$ constraining the RNA then the solvent, and finally equilibrated by slowly releasing the constraints. SHAKE was applied to all hydrogen bonds in the system. The pressure was maintained at 1.0 Pa using Berendsen algorithm [45], and a periodic boundary condition was imposed. A production simulation was performed for $4 \mathrm{~ns}$ with a $2 \mathrm{fs}$ timestep.

The simulations were carried out on SGI-Altix and SGIOrigin computers using eight processors. The analysis for all simulations was performed using the PTRAJ modules on the production simulations excluding the initial equilibration stage. Solvent and sodium ions distribution were analyzed by visual inspection and by evaluation of the most probable atom position at 2-ps intervals in $0.5 \AA^{3}$ resolution grids over a $3.8 \mathrm{~ns}$ trajectory (first 200 ps were omitted from hydration calculations). The most probable positions were estimated by requiring at least an $80 \%$ occupancy of a particular atom of interest within a $0.5 \AA^{3}$ grid element [47].

\subsection{Structural analysis}

Groove widths, backbone torsion angles, and local base pair parameters (twist, tilt, roll, shift, slide, and rise) for each strand and stem were analyzed by the program CURVES 5.1 [48] and compared to standard A-RNA, B-DNA, and A-DNA triplex helical parameters. The standard A-RNA, B-DNA, and A-DNA triplex helices were built using Insight $\mathrm{II}^{\mathrm{R}}$. For comparative purposes the strand in the major groove of the A-DNA triplex was removed during analysis.

\section{Results}

\subsection{Starting structure}

The 48-nucleotide RNA pseudoknot in this study includes the vitally important and conserved regions of the pseudoknot domain including P3 (Stem 2), J2b/3 (Loop 1), part of P2b (Stem 1) and J2a/3 (Loop2) domains (Fig. 1). The model pseudoknot includes the exact human sequence of $\mathrm{J} 2 \mathrm{~b} / 3$ loop and P3 stem, shortened sequence of $\mathrm{J} 2 \mathrm{a} / 3$, and shortened and modified sequence of $\mathrm{P} 2 \mathrm{~b}$. $\mathrm{P} 2 \mathrm{~b}$ contains the original base pairs with phylogenetically conserved nucleotides 97-98, 116-118. The modifications of P2b involve base pairs G93:C121 and G94:C120, which are G93:G121 and C94:G120 in the original sequence. This construct was chosen due to its size and available experimental results $[15,18]$. The length and complexity of the molecular dynamics simulations are dependent on the size of the investigated structure, thus this smaller pseudoknot presents an advantage. This minimal pseudoknot and its mutated versions have been extensively investigated using NMR spectroscopy, telomerase activity assays, and thermal denaturation experiments $[15,18]$, which allow us to directly compare our simulated structure with experimental findings. 
The starting 3D structure of the wild-type telomerase RNA pseudoknot was built directly from its secondary structure using the RNA2D3D software which is described in Section 2. The schematic representation of the secondary structure and the 3D starting structure are shown in Fig. 1. The starting pseudoknot consists of two stems and two loops as determined by phylogenetic analysis [8]. No tertiary interactions between loops and stems are included; therefore, the pseudoknot is initially in a relatively open state. After minimization, heating, and equilibration the starting structure is subjected to $56 \mathrm{~ns}$ of unconstrained MD simulations.

\subsection{Molecular dynamics simulations}

During the initial $16 \mathrm{~ns}$ of simulation the total energy of the pseudoknot structure (Fig. 2a) rapidly improves by approximately $200 \mathrm{kcal} / \mathrm{mol}$. After $16 \mathrm{~ns}$ the structure becomes relatively stable and remains stable for the next $40 \mathrm{~ns}$. We conclude that at this time all major folding/rearrangements of the structure are finished. The initial $16 \mathrm{~ns}$ period will hereafter be called the "stabilization period".

The RMSD relative to the first frame of the whole structure, and for Stem 1, Loop 1, Stem 2, and Loop 2 of the wild-type pseudoknot are shown in Fig. 2b-f. The total structure exhibits large, up to $11 \AA$, deviations during the stabilization period. For the last $40 \mathrm{~ns}$ of the trajectory the RMSD standard deviation is $0.2 \AA$ indicating that the RNA settles into a well-defined and stable configuration during the simulation. As expected during the stabilization period both loops undergo significant adjustments (Fig. 2d, f) due to the formation of the tertiary interactions with the stems and the movement of the loop backbones into the energy minimum. Stem 1 undergoes the least refinement during the stabilization period. However, Stem 2 undergoes significant distortions around 5 ns and stabilizes in a new conformation $5 \AA$ away from the initial Stem structure. Examination of Stem 2 reveals that the large RMSD fluctuation is due to partial reassembly of the pseudoknot junction region,

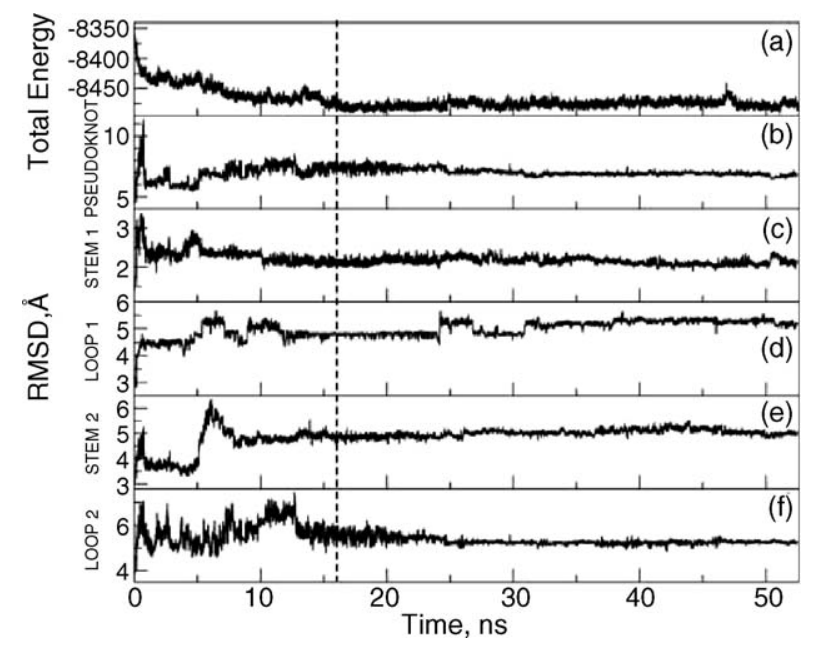

Fig. 2. Total energy and RMSDs compared with the initial frame of the wildtype pseudoknot structure. The vertical dashed line represents the end of the stabilization period. while base pairs in the $3^{\prime}$ side of Stem 2 are stable. To avoid potential artifacts due to implicit solvation we have refined the final wild-type pseudoknot structure with $4 \mathrm{~ns}$ explicit solvent simulations. During $4 \mathrm{~ns}$ the structure exhibited high stability and retained all formed base pairs.

The final structure will be discussed first followed by a discussion of the initial stabilization period. To highlight the effect of the bulge in the wild-type pseudoknot structure we will compare our structure to the NMR solution structure of the $\Delta \mathrm{U} 177$ pseudoknot.

\subsection{Final wild-type pseudoknot structure}

The final structure of the wild-type pseudoknot is welldefined and consists of two stems, two loops and a junction region. The junction region consists of U99-U103, C112U115, and A174-G178. The observed junction region is unique and highly unusual and does not fit into the helical nature of either of the stems and, therefore, will be described as a separate region. The schematic diagram of the most probable tertiary interactions in the final structure together with the stereo snapshots of the lowest energy structure are shown in Fig. 3. Table 1 reflects hydrogen bond occupancies, average distances, and average angles for all Watson-Crick and non-WatsonCrick base pairs retained during the $40 \mathrm{~ns}$ simulation time. The overall global position of the loops and stems are as follows: Loop 2 lies in the minor groove of Stem 1 and Loop 1 lies in the major groove of Stem 2 . The stems rotate at the junction region so that the two loops lie on the same side. This global orientation of the pseudoknot loops and stems is in agreement with the experimental NMR observation of the pseudoknot without the U177 bulge [18]. This configuration of the pseudoknot is stable and the described hydrogen bonds in Fig. 3a are well maintained over the simulation trajectory as can be seen from Table 1.

To further validate our final structure we conducted a set of melting simulations, which were directly compared to UV denaturation experiments [15]. The wild-type pseudoknot profiles show that the tertiary interactions unfold first, followed by Stem 2, and then Stem 1. These results and relative difference between motifs melting temperatures are consistent with the unfolding pathway for the wild-type pseudoknot determined by analysis of the experimental melting profiles [15].

\subsection{Stem 1 and Loop 2 interactions}

Stem 1 and Loop 2 form a stable triple helix with a welldefined structure. Stem 1 is the most stable motif in the structure with an RMSD standard deviation of $0.14 \AA$ and consists of six well-established Watson-Crick base pairs, which are consistent with NMR analysis [15,18]. Loop 2 is positioned in the minor groove of Stem 1 and participates in four triple base interactions with Stem 1 (Fig. 3a). A167 and A168 are rotated slightly outward from the helix, and A173 is tucked into the minor groove and almost parallel to the helical axis without any specific contacts. The curvature and groove 


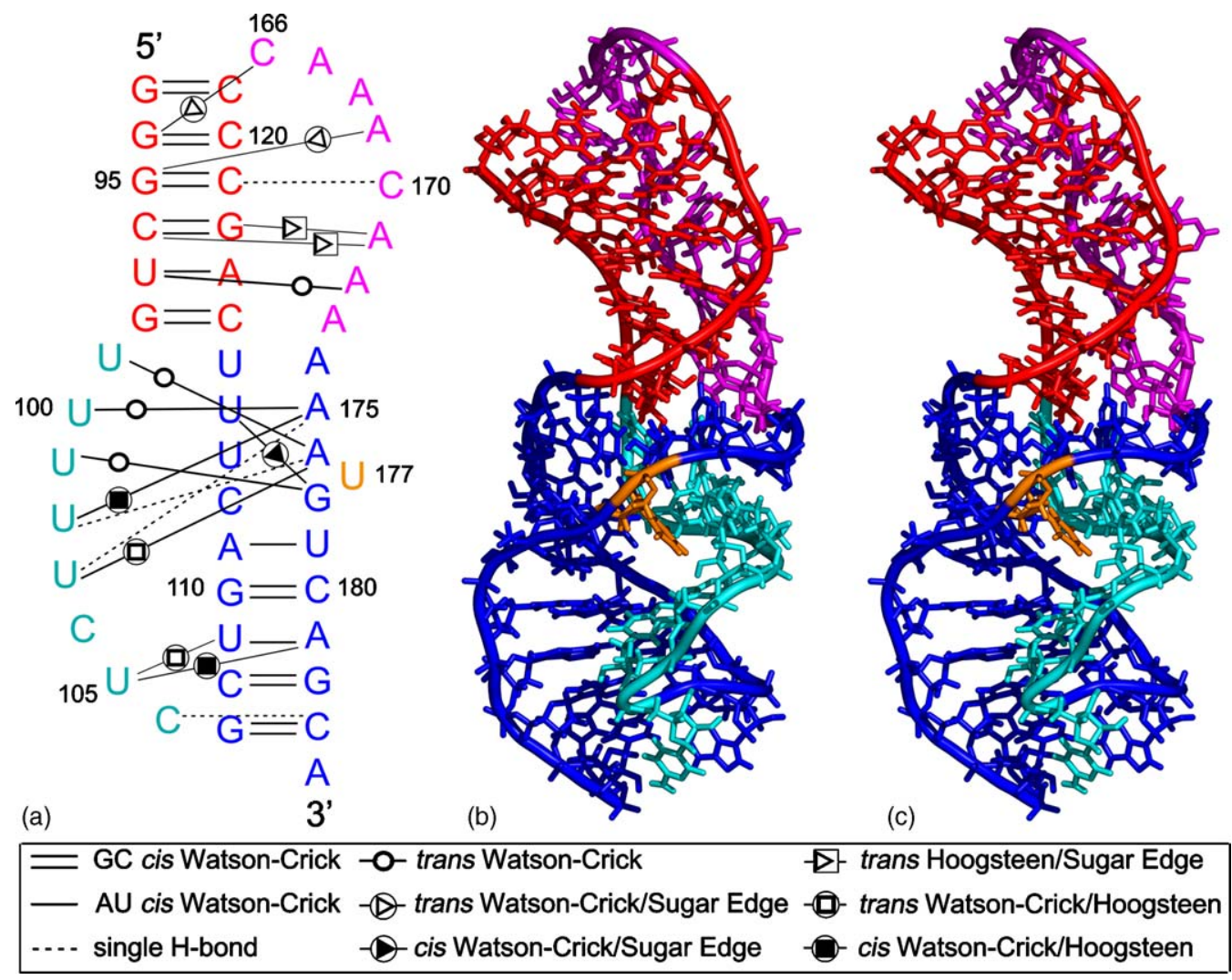

Fig. 3. Final structure of the wild-type pseudoknot which resulted from $56 \mathrm{~ns}$ of molecular dynamics simulations. Shown are the secondary structure with tertiary interactions and the stereo snapshot of the lowest energy structure. The highest occupancy tertiary interactions are marked according to the proposed geometric nomenclature [55].

parameters of Stem 1 resemble the properties of a standard A-DNA triplex without the strand located in the major groove rather than a standard A-RNA. Stem 1 has a wide minor groove, where Loop 2 is located, and a wide major groove with a shallow groove depth equal to $0.5 \AA$ ( $7.44 \AA$ in a standard ADNA triplex). The wide major groove of Stem 1 raises the possibility for other RNA/protein bindings. Stem's 1 global rise $(3.53 \AA)$, roll $\left(11.22^{\circ}\right)$ and twist $\left(39.9^{\circ}\right)$ are approximately equal to those found in A-RNA and Stem's 1 tilt (6.75) is similar to an A-DNA triplex.

The structure and sequence of Stem 1/Loop 2 are the same as the $\Delta \mathrm{U} 177$ pseudoknot structure, thus, we can directly compare this region from our structure with the NMR solution structure of the $\Delta \mathrm{U} 177$ pseudoknot. The RMSDs between the simulated average structure and the NMR $\Delta \mathrm{U} 177$ structure is equal to $1.4 \AA$ for Stem 1 and is equal to $3.17 \AA$ for Loop 2 . However, the majority of the Loop 2 residues A168-A173 produce a smaller RMSD of $2.4 \AA$, where most of the discrepancy comes from the turn of the strand at C166 and A167. Overall, this part of the pseudoknot is in good agreement with the $\Delta \mathrm{U} 177$ NMR solution pseudoknot.

\subsection{Stem 2 and Loop 1 interactions}

Stem 2 is located at the $3^{\prime}$ end of the pseudoknot and consists of five well-defined Watson-Crick base pairs. Loop 1 is positioned in the major groove of Stem 2 and consists of three residues C104-C106. U105 participates in a triple WatsonCrick/Hoogsteen interaction with U109 and A181 of Stem 2. Structural calculations show that the parameters of Stem 2 also resemble an A-DNA triplex with a wide minor groove $(10.2 \AA)$ and a wide $(11.1 \AA)$ and deep $(4.5 \AA)$ major groove where Loop 1 is located.

Superposition of this region of the wild-type pseudoknot and the $\Delta$ U177 solution pseudoknot shows an RMSD of $1.9 \AA$ which is also in a good agreement.

\subsection{Junction region}

Adenine bases are simultaneously able to engage in WatsonCrick and Hoogsteen base pairs. Based on the sequence, the pseudoknot junction should tend to form a triple helix, since the $\mathrm{U}$ rich Loop 1 is stacked in the major groove of Stem 2 which contains A:U base pairs. Ideally, the extra U-rich strand should be involved in Hoogsteen-type base pairs with the A-chain. This will happen only if the helical axis is in the center of the base-triplet [49].

There are indeed base-triplets in the junction region, but not the ones that are expected from the starting structure. The original Watson-Crick base pairs have dissipated and new triple interactions have emerged. As in the $\Delta \mathrm{U} 177$ pseudoknot the junction region of the wild-type pseudoknot is stabilized by triple interactions and uridines 99-102 from Loop 2 are all involved in base pairing with the Stem 2 adenines (175-176) 
Table 1

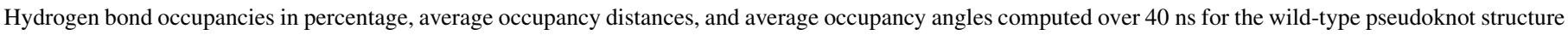

\begin{tabular}{|c|c|c|c|c|c|}
\hline Base pair & Annotation & Hydrogen bond & Occupancy (\%) & Average distance $(\AA)$ & Average angle $\left({ }^{\circ}\right)$ \\
\hline \multicolumn{6}{|l|}{ Stem 1/Loop 2} \\
\hline \multirow[t]{3}{*}{ G93:C121 } & \multirow[t]{3}{*}{ GC cis W.-C. } & H1(G93) $\cdots$ N3(C121) & 99.9 & $3.0(0.1)$ & $18.0(9.1)$ \\
\hline & & $\mathrm{H} 21(\mathrm{G} 93) \cdots \mathrm{O} 2(\mathrm{C} 121)$ & 99.7 & $3.0(0.2)$ & $17.6(9.1)$ \\
\hline & & H41(C121) ‥ O6(G93) & 99.0 & $3.0(0.2)$ & $18.6(10.5)$ \\
\hline \multirow[t]{3}{*}{ G94:C120 } & \multirow[t]{3}{*}{ GC cis W.-C. } & $\mathrm{H} 1(\mathrm{G} 94) \cdots \mathrm{N} 3(\mathrm{C} 120)$ & 99.9 & $3.0(0.1)$ & $15.9(8.5)$ \\
\hline & & H21(G94) $\cdots$ O 2(C120) & 100.0 & $2.9(0.1)$ & $16.9(9.0)$ \\
\hline & & $\mathrm{H} 41(\mathrm{C} 120) \cdots \mathrm{O} 6(\mathrm{G} 94)$ & 99.3 & $3.0(0.2)$ & $17.2(9.5)$ \\
\hline \multirow[t]{2}{*}{ Triple G94:C166 } & \multirow[t]{2}{*}{ CG trans W.-C. /S. } & H22(G94) $\cdots$ N3(C166) & 65.5 & $3.5(0.3)$ & 21.7(11.1) \\
\hline & & $\mathrm{H} 41(\mathrm{C} 166) \cdots \mathrm{N} 3(\mathrm{G} 94)$ & 28.4 & $3.3(0.3)$ & $42.9(13.5)$ \\
\hline \multirow[t]{3}{*}{ G95:C119 } & \multirow[t]{3}{*}{ GC cis W.-C. } & 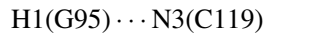 & 99.1 & $3.1(0.2)$ & $20.0(10.4)$ \\
\hline & & H21(G95) ‥ O2(C119) & 100.0 & $2.9(0.1)$ & $16.7(8.7)$ \\
\hline & & H41(C119) ‥ O6(G95) & 88.3 & $3.3(0.3)$ & $18.6(10.0)$ \\
\hline \multirow[t]{2}{*}{ Triple G95:A169 } & \multirow[t]{2}{*}{ AG trans W.-C. /S. } & H22(G95) ‥N1(A169) & 99.6 & $3.1(0.2)$ & $17.5(9.1)$ \\
\hline & & H61(A169) ‥N3(G95) & 30.4 & $3.2(0.2)$ & $45.3(11.4)$ \\
\hline \multirow[t]{3}{*}{ C96:G118 } & \multirow[t]{3}{*}{ CG cis W.-C. } & $\mathrm{H} 1(\mathrm{G} 118) \cdots \mathrm{N} 3(\mathrm{C} 96)$ & 90.3 & $3.2(0.2)$ & $18.2(12.1)$ \\
\hline & & $\mathrm{H} 21(\mathrm{G} 118) \cdots \mathrm{O} 2(\mathrm{C} 96)$ & 72.4 & $3.6(0.2)$ & 19.8(14.9) \\
\hline & & H41(C96) ‥ O6(G118) & 97.5 & $3.0(0.2)$ & 21.9(11.6) \\
\hline Triple C96:A171 & CA trans H./S. & H62(A171) ‥ O2(C96) & 79.7 & $3.0(0.2)$ & $39.0(13.5)$ \\
\hline G118:A171 & GA trans H./S. & $\mathrm{H} 22(\mathrm{G} 118) \cdots \mathrm{O} 2{ }^{\prime}(\mathrm{A} 171)$ & 76.6 & $3.1(0.3)$ & $36.6(12.0)$ \\
\hline \multirow[t]{2}{*}{ U97:A117 } & \multirow[t]{2}{*}{ UA cis W.-C. } & H3(U97) $\cdots$ N1(A117) & 90.8 & $3.1(0.2)$ & $18.9(10.2)$ \\
\hline & & H61(A117) ‥ O4(U97) & 87.2 & $3.2(0.3)$ & $24.9(12.9)$ \\
\hline Triple U97:A172 & UA trans W.-C. & H61(A172) ‥ O2(U97) & 89.2 & $2.9(0.2)$ & $35.5(12.5)$ \\
\hline \multirow[t]{3}{*}{ G98:C116 } & \multirow[t]{3}{*}{ GC cis W.-C. } & H1(G95) ․ N3(C119) & 99.4 & $3.0(0.1)$ & $18.7(9.9)$ \\
\hline & & H21(G95) ‥ O2(C119) & 99.8 & $3.0(0.2)$ & $16.6(8.6)$ \\
\hline & & $\mathrm{H} 41(\mathrm{C} 119) \cdots \mathrm{O}(\mathrm{G} 95)$ & 94.3 & $3.0(0.2)$ & $18.7(10.5)$ \\
\hline Junction & & & & & \\
\hline U99:A176:U103 & AU trans W.-C. & H61(A176) ‥ O2(U99) & 99.8 & $3.0(0.2)$ & $19.0(10.6)$ \\
\hline & & H3(U99) ‥N1(A176) & 99.6 & $3.1(0.2)$ & 18.6(10.5) \\
\hline & UA trans W.-C./H. & H3(U103) ‥ O2P(A176) & 98.9 & $2.9(0.2)$ & $22.1(10.1)$ \\
\hline & & H5(U103) N. N7(A176) & 61.8 & $3.7(0.2)$ & $46.2(7.7)$ \\
\hline & & H8(A176) ‥ O4(U103) & 8.6 & $3.3(0.2)$ & $53.9(6.4)$ \\
\hline A176:U102 & AU cis H./W.-C. & H62(A176) ‥ O4(U102) & 99.1 & $2.9(0.2)$ & $24.2(12.0)$ \\
\hline U100:A175:U102 & UA trans W.-C & H61(A175) $\cdots$ 04(100) & 99.2 & $3.0(0.2)$ & $22.6(10.8)$ \\
\hline & UA $c i s$ W.-C./H. & H3(U102) ‥N7(A175) & 97.1 & $3.3(0.2)$ & $20.9(9.8)$ \\
\hline & & H8(A175) ‥02(102) & 18.1 & $3.4(0.2)$ & $52.9(6.0)$ \\
\hline A175:U103 & AU trans H./W.-C. & H8(A175) ‥ O4(U103) & 91.0 & $3.6(0.2)$ & $28.3(12.6)$ \\
\hline U101:G178:U114 & GU trans W.-C. & H1(G178) ․ O4(U101) & 96.9 & $3.1(0.3)$ & $30.1(11.7)$ \\
\hline & & $\mathrm{H} 21(\mathrm{G} 178) \cdots \mathrm{O} 4(\mathrm{U} 101)$ & 95.1 & $3.1(0.3)$ & $32.6(12.3)$ \\
\hline & UG $c i s$ W.-C./S. & $\mathrm{H} 22(\mathrm{G} 178) \cdots \mathrm{O} 4(\mathrm{U} 114)$ & 93.0 & $2.9(0.2)$ & $20.0(11.3)$ \\
\hline & & H3(U114) ․N3(G178) & 63.0 & $3.7(0.2)$ & $39.8(12.3)$ \\
\hline Stem 2/Loop 1 & & & & & \\
\hline A111:U179 & AU cis W.-C. & H3(U179) $\cdots$ N1(A111) & 94.7 & $3.0(0.2)$ & $20.9(12.0)$ \\
\hline & & H61(A111) ‥ O4(U179) & 89.9 & $3.2(0.3)$ & $18.0(10.0)$ \\
\hline G110:C180 & GC cis W.-C. & $\mathrm{H} 1(\mathrm{G} 110) \cdots \mathrm{N} 3(\mathrm{C} 180)$ & 99.9 & $3.0(0.1)$ & $16.3(8.4)$ \\
\hline & & $\mathrm{H} 21(\mathrm{G} 110) \cdots \mathrm{O} 2(\mathrm{C} 180)$ & 100.0 & $2.9(0.2)$ & $16.3(8.5)$ \\
\hline & & $\mathrm{H} 41(\mathrm{C} 180) \cdots \mathrm{O} 6(\mathrm{G} 110)$ & 99.1 & $3.1(0.2)$ & $17.4(9.6)$ \\
\hline U109:A181 & UA cis W.-C. & H3(U1O9) $\cdots$ N1(A181) & 99.7 & $3.0(0.2)$ & $17.7(9.9)$ \\
\hline & & H61(A181) ‥ O4(U109) & 98.8 & $3.1(0.2)$ & $16.7(9.4)$ \\
\hline Triple A181:U105 & UA $c i s$ W.-C./H. & H62(A181) ‥ O2(U105) & 41.3 & $3.3(0.3)$ & $45.0(10.2)$ \\
\hline U109:U105 & UU cis W.-C./H. & H3(U105) ‥ O4(U109) & 28.2 & $3.2(0.3)$ & 42.1(12.8) \\
\hline & & H5(U109) ․ O4(U105) & 24.0 & $3.7(0.2)$ & $43.6(9.1)$ \\
\hline C108:G182 & CG cis W.-C. & $\mathrm{H} 1(\mathrm{G} 182) \cdots \mathrm{N} 3(\mathrm{C} 108)$ & 99.1 & $3.0(0.1)$ & $16.7(9.0)$ \\
\hline & & $\mathrm{H} 21(\mathrm{G} 182) \cdots \mathrm{O} 2(\mathrm{C} 108)$ & 100.0 & $2.9(0.1)$ & $17.2(9.2)$ \\
\hline & & H41(C108) ‥ O6(G182) & 95.9 & $3.0(0.2)$ & $18.0(10.4)$ \\
\hline G107:C183 & GC cis W.-C. & $\mathrm{H} 1(\mathrm{G} 1 \mathrm{O} 7) \cdots \mathrm{N} 3(\mathrm{C} 183)$ & 73.2 & $3.6(0.2)$ & 49.3(10.2) \\
\hline & & $\mathrm{H} 21(\mathrm{G} 107) \cdots \mathrm{O} 2(\mathrm{C} 183)$ & 82.2 & $3.6(0.3)$ & $44.4(8.0)$ \\
\hline & & H41(C183) ‥ O6(G107) & 42.6 & $3.7(0.3)$ & $25.1(10.6)$ \\
\hline Triple C183:C106 & $\mathrm{CC}$ trans $\mathrm{H} . / \mathrm{H}$. & $\mathrm{H} 42(\mathrm{C} 183) \cdots \mathrm{O} 1 \mathrm{P}(\mathrm{C} 106)$ & 90.4 & $2.9(0.2)$ & $29.5(10.9)$ \\
\hline
\end{tabular}

The maximum allowable hydrogen bond length is $3.8 \AA$. 

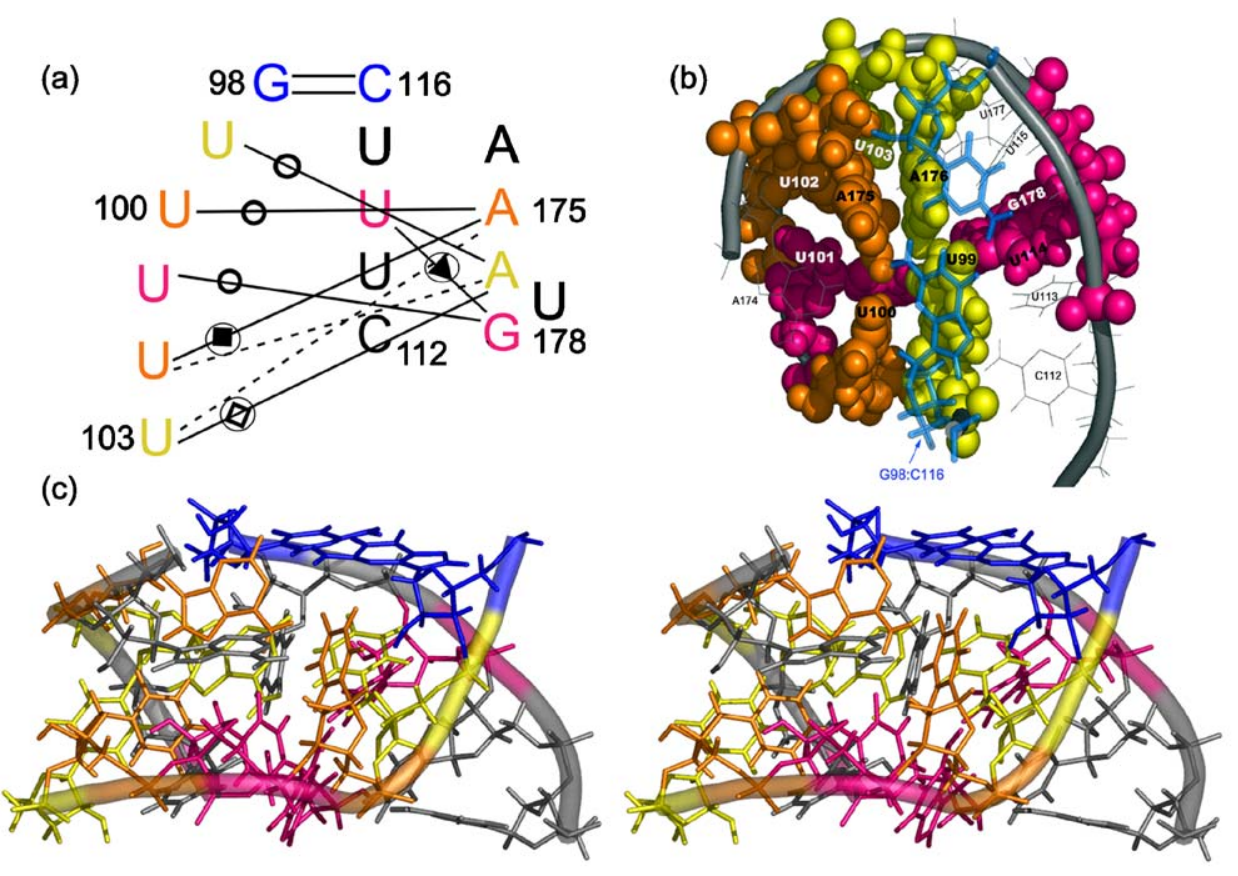

(d)

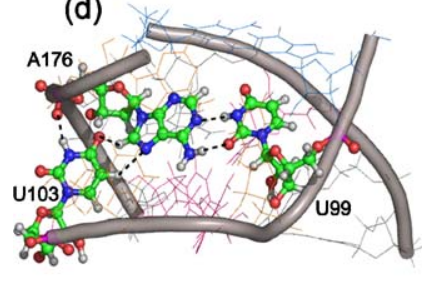

(e)

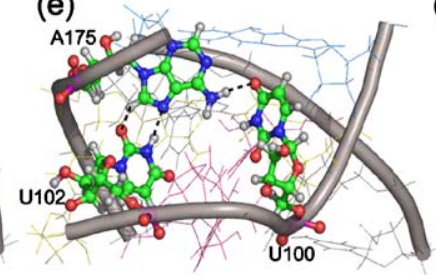

(f)

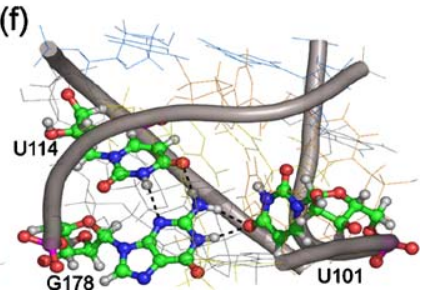

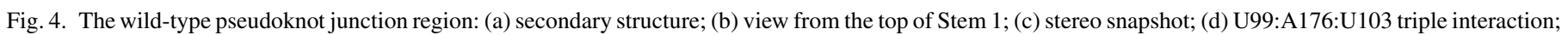

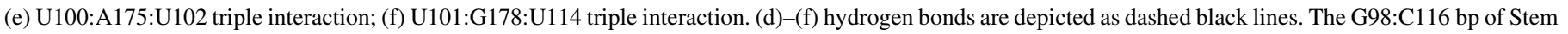
1 is included for comparison.

[18]. However, as depicted in Fig. 4a the base pair stacking in the junction region is highly unusual and quite different from the $\Delta \mathrm{U} 177$ junction region. The U99-U101 bases are connected via trans Watson-Crick base pairs with A175, A176, and G178. U102, U103, and U114 are participating in Watson-Crick/ Hoogsteen or sugar-edge interactions with these base-pairs. A view from the $5^{\prime}$ end of the structure (Fig. $4 \mathrm{~b}$ ) reveals that the U100:A175:U102 triplex stacks over U99:A176:U103, however, these triplexes are almost perpendicular to the stacking of Stem 1 and Stem 2. Another triplex U101:G178:U114 is located almost perpendicular to the UAU triplexes.

Bending, twisting, and translation of each residue in each strand in the junction region play an important role in determining the final structure of pseudoknot. In order to fully describe the junction region we examine the rise, shift, slide, twist, roll, tilt for each strand, and the backbone torsion angles of the residues. The torsion angles are presented in Table 2 . There is a significant change in the strand between residues U115 and C116. This is illustrated by a large negative shift of $-10.2 \AA$, a large negative twist of $-105.1^{\circ}$, and a large positive roll of $66.7^{\circ}$ of U115. This is accompanied by unusual helical values of the $\alpha, \gamma$, and $\xi$, torsion angles of U115. Together this indicates that there is a large kink in the stem and a bend of
U115 into the junction region. Since U114 through G107 are nicely stacked under U115, the kink is required in order to accommodate Stem 2 formation and stability. A174-A176 are also stacked, however, the U177 bulge is rotated outward from the strand into the major groove and is stabilized via hydrogen bond interactions with the $2^{\prime}$-hydroxyl group of U103 securing the global position of Loop 1. U177 has unusual $\alpha, \beta$, and $\gamma$, torsion angles and $\chi$ is in syn conformation. There is a large negative tilt between G178 and U179 which makes possible the stable formation of the G178:U114 bp. Now the distance between the strands with A174-A176 and U113-U115, which formed base pairs in the starting structure, is too small to participate in Watson-Crick interactions. U99 of Loop 1 is stacked under G98, and U100 is stacked under U99, which allows for trans Watson-Crick pairings with A175 and A176. The change in strand direction between U99 and U100 can be illustrated by the unusual $\delta, \varepsilon$, and $\zeta$, torsion angles of U99 and the $\alpha$, and $\gamma$ torsion angles of U100. U101 exhibits a large negative twist of $-153^{\circ}$, a large negative roll of $-83.6^{\circ}$, and a large positive tilt of $73.8^{\circ}$ due to rotation of the strand between U100 and U101. This also can be illustrated by the atypical $\varepsilon$ angle of U100 which is equal to $-105.4^{\circ}$. Large negative roll and positive tilt open the major groove and compress the minor 
Table 2

Torsion angles of standard helical A-RNA, B-DNA, A-DNA triplex, and average torsion angles of residues in the junction region of the wild-type pseudoknot computed over $40 \mathrm{~ns}$

\begin{tabular}{lrrrrrr}
\hline Residue & $\alpha \mathrm{P}-\mathrm{O}_{5}{ }^{\prime}$ & $\beta \mathrm{O}_{5}{ }^{\prime}-\mathrm{C}_{5}{ }^{\prime}$ & $\gamma \mathrm{C}_{5}{ }^{\prime}-\mathrm{C}_{4}{ }^{\prime}$ & $\delta \mathrm{C}_{4}{ }^{\prime}-\mathrm{C}_{3}{ }^{\prime}$ & $\varepsilon \mathrm{C}_{3}{ }^{\prime}-\mathrm{O}_{3}{ }^{\prime}$ & $\zeta \mathrm{O}_{3}{ }^{\prime}-\mathrm{P}$ \\
\hline A-RNA & -62.1 & 180.1 & 47.4 & 83.4 & -151.7 & -73.6 \\
B-DNA & -46.8 & -146 & 36.4 & 156.4 & 155 & -95.2 \\
A-DNA triplex & -49.1 & 178.6 & 40.5 & 82.8 & -163.2 & -66.2 \\
& -47.7 & 171.7 & 42.7 & 82.8 & -155.1 & -75 \\
& -62.8 & 186.2 & 46.9 & 83.1 & -168 & -60.6 \\
Strand 1 & & & & & & \\
U99 & -64 & 170.3 & 69.0 & $\mathbf{1 3 1 . 0}$ & $-\mathbf{7 6 . 9}$ & $\mathbf{7 0 . 4}$ \\
U100 & $\mathbf{7 4 . 2}$ & 195.5 & $\mathbf{1 8 0 . 5}$ & 87.5 & $-\mathbf{1 0 5 . 4}$ & -57.1 \\
U101 & -70.1 & 176.0 & 54.7 & 86.7 & -172.2 & -83.2 \\
U102 & -86.6 & 178.4 & 53.5 & 83.8 & -152.6 & -59.2 \\
U103 & $-\mathbf{1 0 4 . 2}$ & 174.7 & 53.6 & $\mathbf{1 3 4 . 1}$ & $-\mathbf{1 1 2 . 9}$ & -70.0 \\
Strand 2 & & & & & & \\
C112 & -72.8 & 177.2 & 63.0 & 74.1 & -170.9 & -70.8 \\
U113 & $\mathbf{1 4 9 . 1}$ & 187.4 & $\mathbf{1 8 8 . 7}$ & 76.5 & -157.6 & -81.2 \\
U114 & -73.1 & 168.6 & 64.1 & 83.6 & -168.6 & -73.1 \\
U115 & $\mathbf{1 8 1 . 8}$ & 184.1 & $\mathbf{1 4 4 . 7}$ & 80.0 & -171.3 & $\mathbf{8 9 . 2}$ \\
Strand 3 & & & & & & \\
A174 & -75.2 & 176.9 & 56.9 & $\mathbf{1 3 0 . 9}$ & $-\mathbf{8 6 . 5}$ & $\mathbf{6 6 . 2}$ \\
A175 & $\mathbf{8 2 . 2}$ & 191.0 & $\mathbf{1 8 7 . 2}$ & 83.7 & -138.6 & -55.8 \\
A176 & -84.4 & 175.7 & 56.8 & 79.1 & -129.8 & -95.7 \\
U177 & $-\mathbf{1 2 8 . 6}$ & $\mathbf{1 1 4 . 7}$ & $\mathbf{1 9 3 . 3}$ & 79.2 & -170.6 & -83.9 \\
G178 & -72.9 & 176.1 & 56.4 & 82.6 & -169.4 & -83.9 \\
\hline Bold font & & & & & & \\
\hline
\end{tabular}

Bold font is used to highlight unusual angles.

groove, which allows U101 to participate in a trans WatsonCrick base pair with G178, and U102 and U103 to participate in Hoogsteen interactions with the U100:A175 and U99:A176 bp, respectively. There is another kink in the strand between U103 and $\mathrm{C} 104$, which corresponds to a change in the $\delta$ and $\varepsilon$ torsion angles of U103. This kink allows C104-C106 to be tucked into the major groove of Stem 2.

\subsection{Explicit solvent refinement}

Simulations with explicit water show that the final structure is stable and that formed base pairs are maintained (Fig. 5a). The most probable hydration sites (red dotted spheres) and sodium ion locations (blue spheres) are shown in Fig. 5b. The yellow spheres represent the most occupied (more than 90\%) water positions. The sodium ions are found in the open major groove of Stem 1 which is expected according to previous studies [47]. However, no ion retention is observed in the major groove of Stem 2 possibly since it is occupied by Loop 1 . The condensation of the water around and inside the junction is apparent which indicates that the junction region is stable and relatively rigid [47]. The most occupied water position inside the junction (yellow sphere) indicates the water insertion between the backbone and U114. Overall, no water inserted base pairs were observed.

\subsection{Initial folding/stabilization period}

To understand the reason for the breaking of the initial basepairing in the junction, the stabilization period was examined (a)

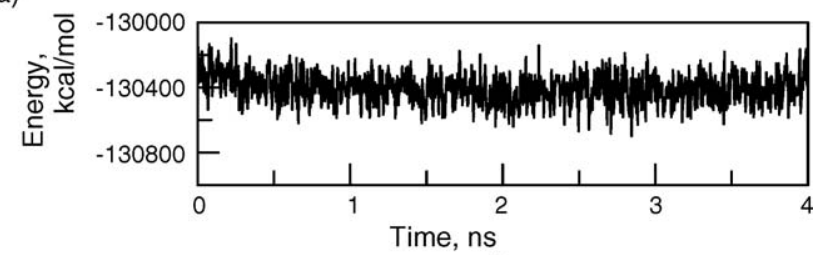

(b)

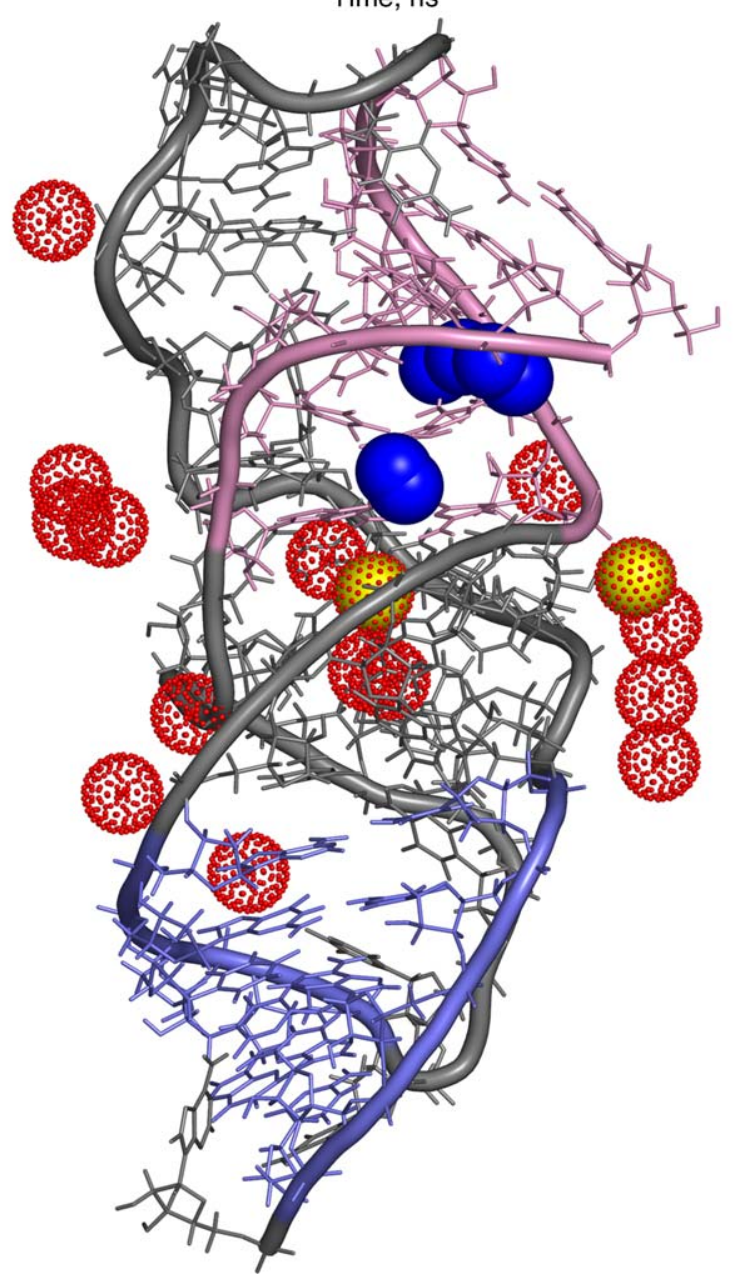

Fig. 5. (a) Potential energy of the wild-type pseudoknot structure in explicit solvent; (b) hydration of the wild-type pseudoknot structure. Red spheres represent the most probable hydration sites and yellow spheres symbolize the most highly occupied positions of water. Blue solid spheres correspond to the most probable counterion locations. Stem 1 residues are colored pink and Stem 2 residues are colored light blue.

in detail. The hydrogen-bond distances between the starting base pairs U115:A174, U114:A175, U113:A176, and C112: G178 are shown in Fig. 6. The interactions between the bases closest to the junction U115:A174 (Fig. 6a) are lost after 200 ps due to the temporal formation of the U99:A174 Watson-Crick/ Hoogsteen base pair (Fig. 7b). The base pair U114:A175 (Fig. 6b) becomes unstable at the same time and its interactions are lost until $2.2 \mathrm{~ns}$. However, these bases remain aligned for bonding and do indeed reform hydrogen bonds from 2.2 ns until 3.5 ns. The U113:A176 and C112:G178 (Fig. 6c, d) are stable until about the 5 ns simulation time. The C112:G178 regains stability afterwards; however, at around $10 \mathrm{~ns}$ it separates for the rest of the simulation. Therefore, the plots 


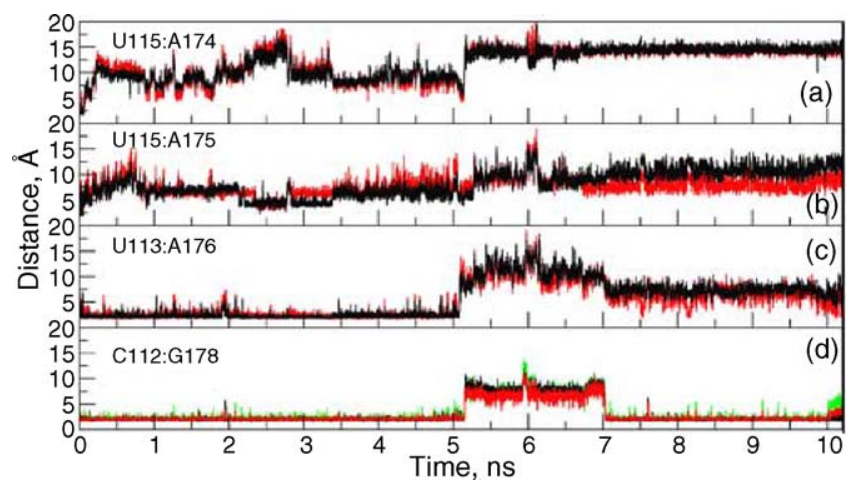

Fig. 6. Plot of the Watson-Crick hydrogen bond distances between starting base pairs in the junction region vs. time during first $10.2 \mathrm{~ns}$ of the stabilization period: (a) U115:A174; (b) U114:A175; (c) U113:A176; (d) C112:G178. For AU base pairs the distance between $\mathrm{H} 61(\mathrm{~A})$ and $\mathrm{O} 4(\mathrm{U})$ is black, and the distance between $\mathrm{H} 3(\mathrm{U})$ and N1(A) is red. For the GC base pair, H41(C) and O6(G) distance is black, $\mathrm{H} 1(\mathrm{G})$ and $\mathrm{N} 3(\mathrm{C})$ distance is red, and $\mathrm{H} 21(\mathrm{G})$ and $\mathrm{O} 2(\mathrm{C})$ distance is green.

in Fig. 6 illustrate that the crucial change in the junction region occur at around $5 \mathrm{~ns}$.

The base pair breaking events were observed in connection with the rotation of the $\chi$ A174 glycosyl torsion angle from the anti $\left(-110^{\circ}\right)$ to the high-anti $\left(-40^{\circ}\right)$ conformational region (Fig. 7a). Movements of A174 and its $\chi$ angle are highly correlated with the global RNA motions and the base pair formations in the junction region, suggesting that the degree of conformational freedom in A174 is of great importance for junction refolding and stabilization. The flexibility of A174 is influenced by the degree of its interactions with U99 which can

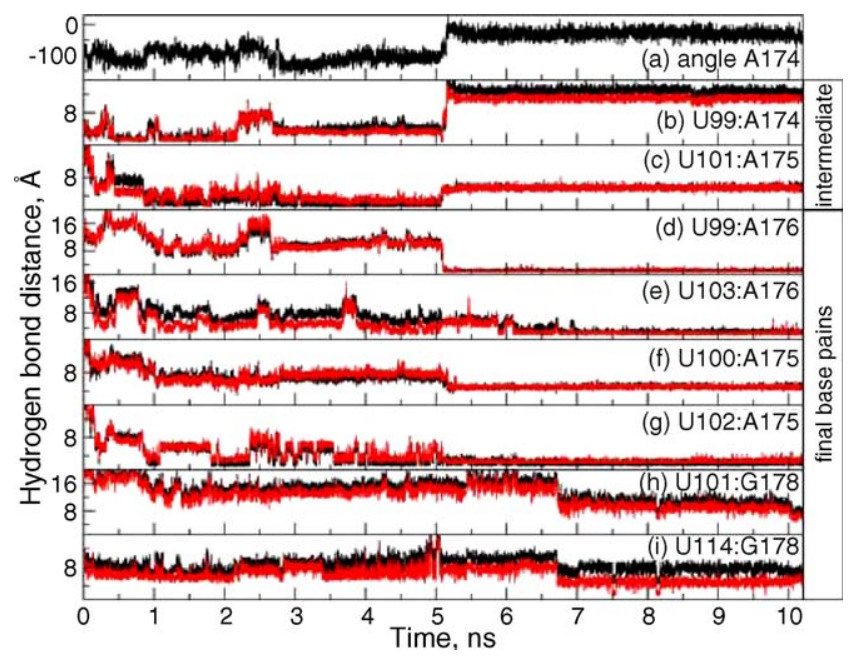

Fig. 7. Dynamics data of the base-pair formations in the junction region during the initial $10.2 \mathrm{~ns}$ of the stabilization period: (a) $\chi$ angle of A174. Hydrogen bond distances of the intermediate Watson-Crick/Hoogsteen base pairs (b) U99:A174; and (c) U101:A175, where red is the distance between H62(A) and $\mathrm{O} 4(\mathrm{U})$ and black is the distance between $\mathrm{H} 3(\mathrm{U})$ and N7(A). Hydrogen bond distances of the final base pairs of the triple interactions (d)-(e) U99:A176:U103; (f)-(g) U100:A175:U102; (h)-(i) U101:G178:U114. (d) H61(A176):O2(U99) is red and N1(A176):H3(U99) is black; (e) N7(A176):H6(U103) is red and H8(A176):O4(U103) is black; (f) H61(A175):O2(U100) is red and N1(A175) :H3(U100) is black; (g) N7(A175):H3(U102) is red and H8(A175):O2(U102) is black; (h) H21(G178):O4(U101) is red and Hl(G178):O4(U101) is black; (i) $\mathrm{H} 22(\mathrm{G} 178): \mathrm{O} 4(\mathrm{U} 114)$ is red and N3(G178):H3(U114) is black. be seen in Fig. 7b where the hydrogen bond distances between U99 (Watson-Crick face) and A174 (Hoogsteen edge) are shown. The U115:A174 bp is lost due to the formation of the intermediate U99:A174 Watson-Crick/Hoogsteen base pair. U101 is involved in an intermediate pairing with the Hoogsteen edge of A175 (Fig. 7c), while A175 is still intermittently bonded to U114. At around $5 \mathrm{~ns}$ both intermediate base pairs, U99:A174 and U101:A175, are broken to accommodate the formation of the other base pair that will be retained throughout the rest of the simulation. U99 moves to pair up with A176 (Fig. 7d), A175 is stabilized by pairing with U102 (Fig. 7g) and moments later with U100 (Fig. 7f) to form the base triple U100:A175:U102. As a consequence residues U113 and U114 move away from the adenines to a distance that is too large to form hydrogen bonds. About 1 ns later U103 hydrogen bonds to A176 (Fig. 7e) which completes another stable triple interaction U99:A176:U103. U114 interacts with G178 via a single hydrogen bond around $6.5 \mathrm{~ns}$ (Fig. 7i), and the U101:G178 (Fig. 7h) interaction stabilizes around $15 \mathrm{~ns}$, when U177 moves into its final position. A174 is $100 \%$ conserved in telomerase RNA. However, U115, that presumably forms a base pair with A174, is not evolutionary conserved and is replaced by a cytosine in some species [8]. Therefore, in these species there is no standard bonding between C115 and A174 and the flexibility of A174 could be even more prominent.

\subsection{Influence of U177 on structure formation}

Interestingly, the formation of the intermediate and the final base pairs of the wild-type pseudoknot is also connected to the interaction of the U177 bulge with the uridines in the Loop 1 strand (Fig. 8). U177 is flexible, and intermittently moves across the major groove interacting with U99, U101, U102 and U103 via formation of unstable noncanonical UU

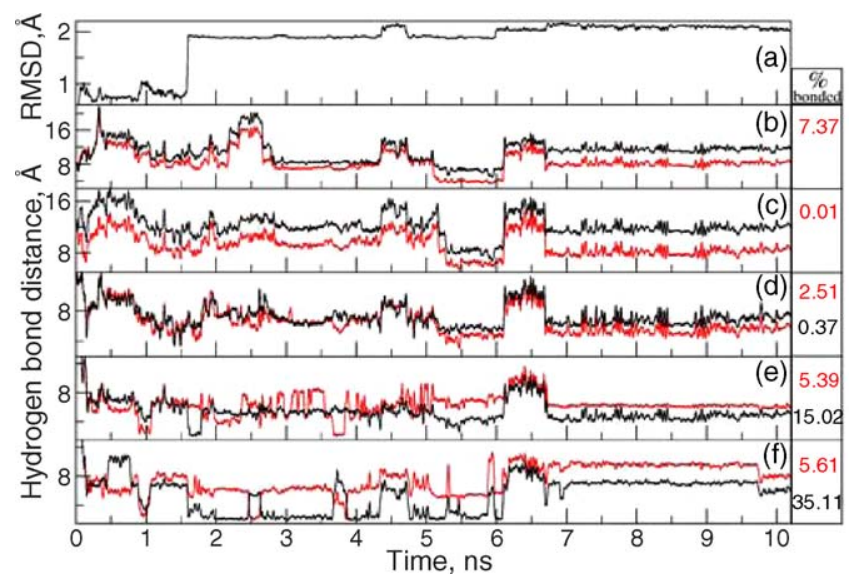

Fig. 8. Interactions of the U177 bulge and the uridine residues in Loop 1 during the $10.2 \mathrm{~ns}$ of the stabilization period. (a) RMSD compared with the starting coordinates of U177. Hydrogen bond distances between U177 bulge and the uridine residues of Loop 1. (b) U99:U177; (c) U100:U177; (d) U101:U177; (e) U102:U177; (f) U103:U177. UU base pair can exist in two conformations (red) and (black). Red is the distance between $\mathrm{H} 3$ (U177) and $\mathrm{O} 4$ (U*) and represents the first conformation, and black is the distance between $\mathrm{H} 3\left(\mathrm{U}^{*}\right)$ and $\mathrm{O} 4(\mathrm{U} 177)$ represents the second conformation. Hydrogen bond occupancies for both conformations for each UU base pair are located on the right. 
base pairs. Uridine bases can form a wobble base pair between the imino hydrogens $\mathrm{H}_{3}$ and $\mathrm{O}_{4}$ of one base and $\mathrm{O}_{2}$ of the other, which can be realized in two symmetrical conformations. A UU base pair can dynamically switch between these two conformations [50]. The hydrogen bond distances and occupancies for both conformations between U177 and U99-U103 for the initial $10 \mathrm{~ns}$ of the simulation are presented in Fig. 8b-f. At the beginning of the simulations U177 is protruding out of the helix into the major groove and periodically interacts with the various uridines in Loop 1. Around $1.5 \mathrm{~ns} \mathrm{U} 177$ rotates around its $\chi$ angle changing its conformation from anti to syn (Fig. 8a), which initiates a stable interaction with U103 (Fig. 8f). Between 4.25 and 4.75 ns the temporal loss of the U103:U177 interactions (Fig. 8f), the U177 RMSD fluctuations (Fig. 8a), and the increase in the distance between U177 and Loop 1 uridines (Fig. 8b-d) are attributed to the complementary change in the U177 torsion angles towards the helical values of standard A-RNA: $\alpha$ from trans to - gauche, $\delta$ from trans to + gauche, $\varepsilon$ from + gauche to - gauche, and $\xi$, from + gauche to - gauche. At around 5 ns the interaction between U177 and U99 (Fig. 8b) assists in the breaking of U113:A176 (Fig. 6c) and the formation of the U99:U176 bp (Fig. 7d). There is also a subsequent temporal change in the U177 torsion angles: $\alpha$ from trans to - gauche and $\beta$ from + gauche to trans. At around $6 \mathrm{~ns}$ the loss of interactions between $\mathrm{U} 177$ and U103 (Fig. 8f) leads to the formation of U103:A176 (Fig. 7e). The loss of U177:U103 interactions accompanied by the significant distance increase between $\mathrm{U} 177$ and all of Loop 1 uridines is caused by the U177 $\delta, \varepsilon$, and $\xi$, torsion angles shifting into the ranges of standard ARNA helical angles. The high distance fluctuations continue until around $6.7 \mathrm{~ns}$ when the switch of the U177 $\alpha$ angle (from + gauche to - gauche) and $\beta$ angle (from trans to + gauche) stabilizes the movement of U177 and consequently the junction residues. Simultaneously the interaction of U177 with U102 (Fig. 8e) assists in hydrogen bond formation of G178 with U114 (Fig. 7i). After 6.7 ns most of the drastic reformation of the junction region is complete, however, minor reorganizations continue until $16 \mathrm{~ns}$.

The final rearrangements of the junction stabilize after U177 goes into its final position by forming an interstrand hydrogen bond with the backbone between U103 and C104 which stabilizes the interactions between the strands. The key function of U177 is to bring the U99-U103 residues into close proximity of Stem 2, which initiates the competition between U113-U115 and U99-U101 for interaction with A174-A176. Therefore, the U177 bulge acts as a critical element that is required for stabilization and rearrangement of the junction region.

\section{Discussion}

A comparison of the average NMR $\Delta \mathrm{U} 177$ solution structure (PDB code 1YMO) and the average simulated wild-type structure of the telomerase RNA pseudoknot shows an overall similar fold with an RMSD value of $4.9 \AA$ excluding the U177 bulge. The RMSD of NMR $\Delta \mathrm{U} 177$ pseudoknot structures to its mean is equal to $1.25 \pm 0.29 \AA$ [18]. The overlay of these two structures indicates that the global positioning of these two pseudoknots is very similar (Fig. 9). Both pseudoknots form an extended triple helix with Loop 2 positioned in the minor groove of Stem 1 and Loop 1 positioned in the major groove of Stem 2. Due to a twist in the junction, both loops are situated on one side of the pseudoknot. There are important triple base tertiary interactions occurring in the junction region in both pseudoknots. The uridines from Loop 1 are all participating in hydrogen bond interactions with adenines in Stem 2. However, the nature of these triple base-pair interactions in the junction is different between the wild-type and the $\Delta \mathrm{U} 177$ pseudoknots. Denaturation experiments also show that the melting temperature of the tertiary interactions of the $\Delta \mathrm{U} 177$ pseudoknot is higher by $11^{\circ}$ than that of the wild-type pseudoknot [18].

Stem 1 and Loop 2 have exactly the same sequence in the wild-type pseudoknot and in the $\Delta \mathrm{U} 177$ pseudoknot. Even though Stem 1/Loop 2 region have a similar overall position and fold with an RMSD of $4.1 \AA$, tertiary interactions between Stem 1 and Loop 2 are different. The difference in tertiary interactions in this region between the wild-type and the $\Delta \mathrm{U} 177$ pseudoknots comes from the difference in the junction region. For example, in the $\Delta \mathrm{U} 177$ pseudoknot U99 interacts with A173 creating Loop 1/Loop 2 interactions, which brings Loop 2 into a slightly different position relative to Stem 1 . In the predicted wild-type pseudoknot structure, U99 interacts with A175 of Stem 2 and A173 does not participate in any base pairings. Thus, different interactions in the junction also lead to different tertiary interactions in the Stem 1/Loop 2 region.

Therefore, the major difference between the wild-type and the $\Delta \mathrm{U} 177$ pseudoknots comes from the junction region, which can be attributed to the existence of the extra bulged nucleotide U177 in our structure, which changes the helical axis of Stem 2 and consequently the interactions between strands. Bulges are known to cause distortions of A-type conformations of RNA helices by changing the global geometry of the structure and creating a twist or a kink in a helical region which can make unusual hydrogen-bonding contacts accessible [20,22-24]. Furthermore, bulges and non-Watson-Crick base pairs are also important for protein recognition and binding and for initiation of RNA tertiary folding [19-21,51]. Indeed, the removal of the U177 bulge from the telomerase RNA pseudoknot stabilizes the structure in the pseudoknot form and prevents it from unfolding into the hairpin form, considerably reducing telomerase activity and functionality [11,18]. Thermodynamic parameters obtained via denaturation experiments also confirm the extra stability gained by the deletion of U177, with a total $\Delta G$ improvement of $4.2 \mathrm{kcal} / \mathrm{mol}[11,18]$. The telomerase pseudoknot structure and especially sequence is critically important for stable in vivo binding of hTERT to hTR [52]. Furthermore, it has been shown that the junction sequence and interactions are crucial for functionality of pseudoknots [53]. For example, the study of the retroviral gag-pro frameshift-stimulating pseudoknots and their derivatives, a pseudoknot from the gene 32 mRNA of bacteriophage T2 that is not naturally associated with frameshifting, and hybrids of these pseudoknots propose that the dynamics of the stem and loop residues at the junction play a role in determining frameshifting efficiency [54]. 


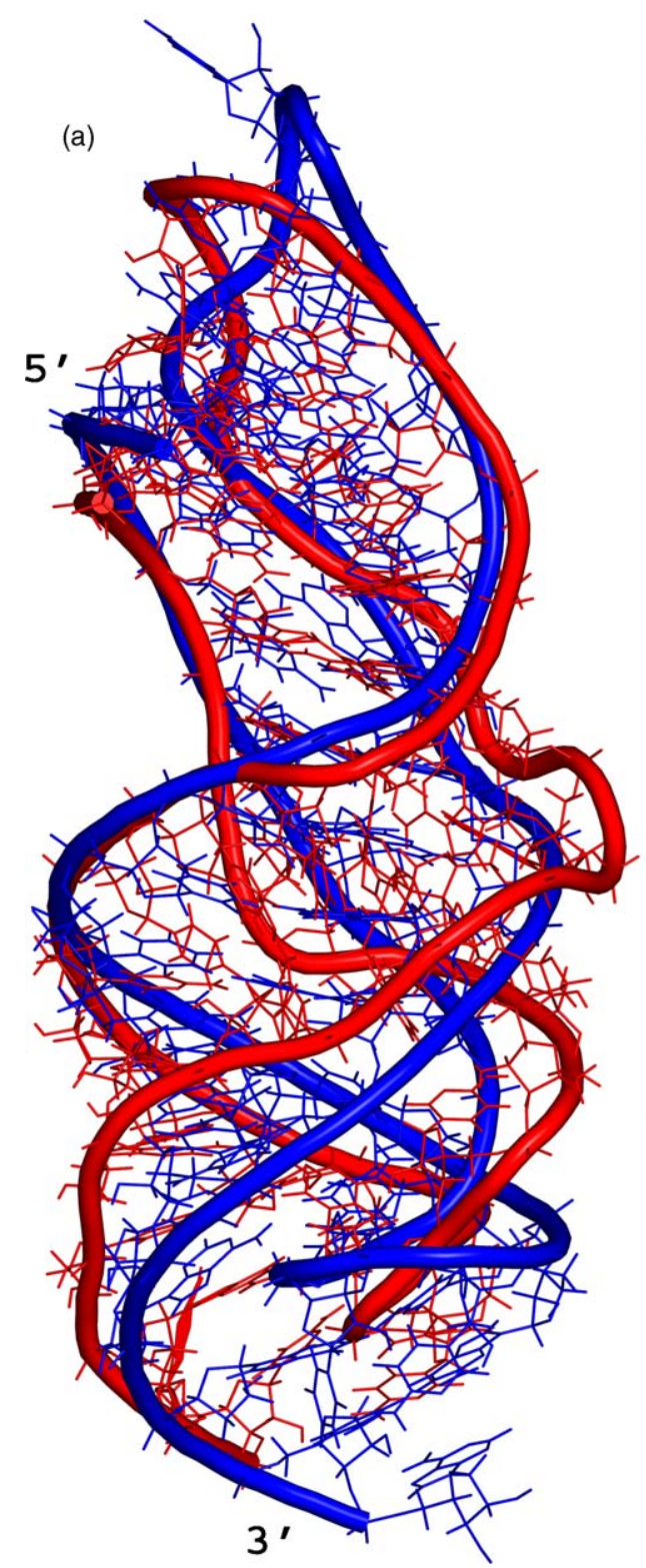
$G=C^{166} A$
(b) $\mathrm{G} \rightleftharpoons \mathrm{C}_{120}$ \&
$95 \mathrm{G}=\mathrm{C} \cdots \cdots \cdots \cdots \cdots \cdot \mathrm{C} \mathrm{C}_{170}$
$\mathrm{C} \equiv \mathrm{G} \triangleright \mathrm{D} \mathrm{A}$
$U=A$ ○A
$G=C \quad A$

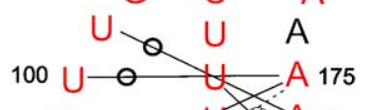

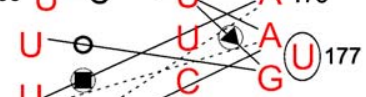<smiles>[Li][B][Ca]</smiles>

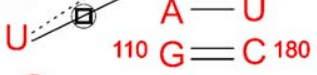

C

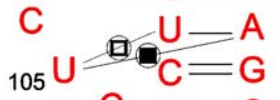

C. $\mathrm{G}=\mathrm{C}$
(c)<smiles>O=C=C1C=CC(C=O)C1</smiles>
$95 \mathrm{G}=\mathrm{C} \mathrm{Cl}_{170}$

$C=G \quad-A$

$U=A \backsim A$

$U=\frac{C}{U-A} A$

$100 \mathrm{U} U \mathrm{U} 175$

$U \longrightarrow U$

$U \rightarrow C=G$

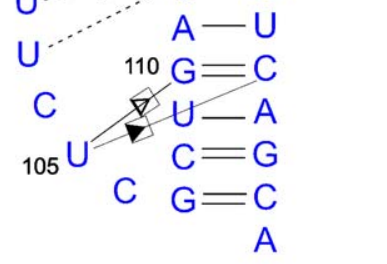

Fig. 9. (a) Superposition of the average wild-type pseudoknot structure (red) and the 1YMO average NMR structure without the U177 bulge (blue). The RMSD between these two structures is equal to $4.9 \AA$. Secondary structures of (b) the wild-type pseudoknot and (c) the average $\Delta$ U177 pseudoknot structure.

Moreover, it has been suggested that the nonframeshifting bacteriophage pseudoknot, which has an average structure that is similar to the frameshifting pseudoknots, does not sample the specific conformations at the junction that are required for frameshifting activity [54]. Also mutational analyses show that the identities of the nucleotides near the junction of the pseudoknot influence the efficiency of the frameshifting pseudoknot [54] and the activity of the telomerase pseudoknot [13]. Since our wild-type pseudoknot and the $\Delta U 177$ pseudoknot have different functionalities and sequences, it is expected that the interactions in the junction region will be different. We propose that the difference in the junction region is related to the difference in the functionality of the wild-type telomerase pseudoknot which acts as a dynamical biological switch compared with the static behavior of the $\Delta \mathrm{U} 177$ pseudoknot. The unusual bonding in the junction of the wild- type pseudoknot could possibly be the reason for its lower stability than the $\Delta \mathrm{U} 177$ pseudoknot and could, therefore, be an important feature which allows the wild-type pseudoknot structure to intermittently switch from the pseudoknot to the hairpin forms.

In summary, the data presented here strongly suggests that the presence of the U177 bulge in the wild-type telomerase is crucial for the interactions and structural formation of the junction region of the pseudoknot. We conclude that the predicted junction interactions assume an important role in the functionality of the telomerase RNA.

We have also applied the same methodology to the study of the effects of the DKC mutations on the pseudoknot structure and formation. We found that DKC-mutations abolish the formation of the P3 helix, change the global orientation and appearance of the pseudoknot, and overall destabilize the 
structure. Overall, our results are consistent with experimental observations and support published biochemical data.

\section{Acknowledgments}

This research was supported by the Intramural Research Program of the NIH, National Cancer Institute, Center for Cancer Research. The computational support was provided by the National Cancer Institute's Advanced Biomedical Computing Center.

\section{References}

[1] C. Autexier, C.W. Greider, Telomerase and cancer: revisiting the telomere hypothesis, Trends Biochem. Sci. 21 (1996) 387-391.

[2] M.P. Granger, W.E. Wright, J.W. Shay, Telomerase in cancer and aging, Crit. Rev. Oncol. Hematol. 41 (2002) 29-40.

[3] J.W. Shay, W.E. Wright, Telomerase: a target for cancer therapeutics, Cancer Cell 2 (2002) 257-265.

[4] J. Feng, W.D. Fung, S. Wang, S.L. Weinrich, A.A. Avilion, C.P. Chiu, R.R Adams, E. Chang, R.C. Allsopp, J. Yu, S. Le, M.D. West, C.B. Harley, W.H. Andrews, C.W. Greider, B. Villeponteau, The RNA component of human telomerase, Science 269 (1995) 1236-1241.

[5] T.C. Leeper, G. Varani, The structure of an enzyme-activating fragment of human telomerase, RNA 11 (2005) 394-403.

[6] H. Yamaguchi, R.T. Calado, H. Ly, S. Kajigaya, G.M. Baerlocher, S.J. Chanock, P.M. Lansdorp, N.S. Young, Mutations in TERT, the gene for telomerase reverse transcriptase, in aplastic anemia, N. Engl. J. Med. 352 (2005) 1413-1424.

[7] T. Vulliamy, A. Marrone, F. Goldman, A. Dearlove, M. Bessler, P.J. Mason, I. Dokal, The RNA component of telomerase is mutated in autosomal dominant dyskeratosis congenital, Nature 413 (2001) 432-435.

[8] J.-L. Chen, M.A. Blasco, C.W. Greider, Secondary structure of vertebrate telomerase RNA, Cell 100 (2000) 503-514.

[9] J.-L. Chen, C.W. Greider, Telomerase RNA structure and function: implications for dyskeratosis congenital, Trends Biochem. Sci. 29 (2004) 183-192.

[10] D. Gilley, E.H. Blackburn, The telomerase RNA pseudoknot is critical for the stable assembly of a catalytically active ribonucleoprotein, PNAS U.S.A. 96 (1999) 6621-6625.

[11] L.R. Comolli, I. Smirnov, L. Xu, E.H. Blackburn, T.L. James, A molecular switch underlies a human telomerase disease, PNAS 99 (2002) 1699817003.

[12] M.A. Cerone, R.J. Ward, J.A. Londono-Vallejo, C. Autexier, Telomerase RNA mutated in autosomal dyskeratosis congenita reconstitutes a weakly active telomerase enzyme defective in telomere elongation, Cell Cycle 4 (2005) 585-589.

[13] H. Ly, E.H. Blackburn, T.G. Parslow, Comprehensive structure-function analysis of the core domain of human telomerase RNA, Mol. Cell. Biol. 23 (2003) 6849-6856.

[14] M. Antal, E. Boros, F. Solymosy, T. Kiss, Analysis of the structure of human telomerase RNA in vivo, Nucleic Acids Res. 30 (2002) 912-920.

[15] C.A. Theimer, L.D. Finger, L. Trantirek, J. Feigon, Mutations linked to dyskeratosis congenital cause changes in the structural equilibrium in telomerase RNA, PNAS 100 (2003) 449-454.

[16] L. Martin-Rivera, M.A. Blasco, Identification of functional domains and dominant negative mutations in vertebrate telomerase RNA using an in vivo reconstitution system, J. Biol. Chem. 276 (2001) 5856-5865.

[17] J.L. Chen, C.W. Greider, Functional analysis of the pseudoknot structure in human telomerase RNA, PNAS 102 (2005) 8080-8085.

[18] C.A. Theimer, C.A. Blois, J. Feigon, Structure of the human telomerase RNA pseudoknot reveals conserved tertiary interactions essential for function, Mol. Cell. 17 (2005) 671-682.

[19] C. Dingwall, I. Ernberg, M.J. Gait, S.M. Green, S. Heaphy, J. Karn, A.D. Lowe, M. Singh, M.A. Skinner, HIV-1 tat protein stimulates transcription by binding to a U-rich bulge in the stem of the TAR RNA structure, EMBO J. 9 (1990) 4145-4153.

[20] T. Hermann, D.J. Patel, RNA bulges as architectural and recognition motifs, Structure 8 (2000) R47-R54

[21] C.R. Woese, R.R. Gutell, Evidence for several higher order structural elements in ribosomal RNA, Proc. Natl. Acad. Sci. 86 (1989) 3119-3122.

[22] M. Feig, M. Zacharias, B.M. Pettitt, Conformations of an adenine bulge in DNA octamer and its influence on DNA structure from molecular dynamics simulations, Biophys. J. 81 (2001) 352-370.

[23] M. Zacharias, P.J. Hagerman, Bulge-induced bends in RNA: quantification by transient electric birefringence, J. Mol. Biol. 247 (1995) 486500 .

[24] Y. Xiong, J. Deng, C. Sudarsanakumar, M. Sundaralingam, Crystal structure of an RNA duplex r(gugucgcac) $)_{2}$ with uridines bulge, J. Mol. Biol. 313 (2001) 537-582.

[25] T.E. Cheatham III, M.A. Young, Molecular deynamics simulations of nucleic acids: successes, limitations, and promise, Biopolymers 56 (2001) 232-256.

[26] M. Zacharias, Simulation of the structure and dynamics of nonhelical RNA motifs, Curr. Opin. Struc. Biol. 10 (2000) 311-317.

[27] E. Giudice, R. Lavery, Simulations of nucleic acids and their complexes, Acc. Chem. Res. 35 (2002) 350-357.

[28] P. Auffinger, E. Westhof, Simulations of the molecular dynamics of nucleic acids, Curr. Opin. Struc. Biol. 8 (1998) 227-236.

[29] P. Auffinger, A.C. Vaiana, in: R.K. Hartmann, A. Bindereif, A. Schon, E. Westhof (Eds.), Molecular dynamics of RNA systems in handbook of RNA biochemistry, Wiley-VCH Verlag GmbH \& Co. KGaA, Weinheim, 2005, pp. 560-576.

[30] T.E. Cheatham III, P.A. Kollman, Molecular dynamics simualtions of nucleic acids, Annu. Rev. Phys. Chem. 51 (2000) 435-471.

[31] M.S. Lee, F.R. Salsbury Jr., C.L. Brooks III, Novel generalized born methods, J. Chem. Phys. 116 (2002) 10606-10614.

[32] M. Feig, A. Onufriev, M.S. Lee, W. Im, D.A. Case, C.L. Brooks III, Performance comparison of generalized born and poisson methods in the calculation of electrostatic solvation energies for protein structures, J. Comp. Chem. 25 (2004) 265-284.

[33] L.Y. Zhang, E. Gallicchio, R.A. Friesner, R.M. Levy, Solvent models for protein-ligand binding: comparison of implicit solvent poisson and surface generalized born models with explicit solvent simulations, J. Comp. Chem. 22 (2001) 591-607.

[34] W. Cornell, R. Abseher, M. Nilges, D.A. Case, Continuum solvent molecular dynamics study of flexibility in interleukin-8, J. Mol. Graph. Model. 19 (2001) 136-145.

[35] D. Bashford, D.A. Case, Generalized born models of macromolecular solvation effects, Ann. Rev. Phys. Chem. 51 (2000) 129-152.

[36] V. Tsui, D.A. Case, Theory and applications of the generalized born solvation model in macromolecular simulations, Biopolymers 56 (2001) 275-291.

[37] J. Weiser, P.S. Shenkin, W.C. Still, Approximate atomic surfaces from linear combinations of pairwise overlaps (LCPO), J. Comp. Chem. 20 (1999) 217-230.

[38] M. Scarsi, J. Apostolakis, A. Caflisch, Comparison of a GB model with explicit solvent simulations: potentials of mean force and conformational preferences of alanine dipeptide and 1,2-dichloroethane, J. Phys. Chem. B 102 (1998) 3637-3641.

[39] J. Srinivasan, J. Miller, P.A. Kollman, D.A. Case, Continuum solvent studies of the stability of RNA hairpin loops and helices, J. Biomol. Struct. Dyn. 16 (1998) 671-682.

[40] G.M. Clore, C.D. Schwieters, Theoretical and computational advances in biomoleular NMR spectroscopy, Curr. Opin. Struct. Biol. 12 (2002) 146153.

[41] H.M. Matinez, J. Maizel Jr., B.A. Shapiro, RNA2D3D. A program for generating, viewing, and comparing 3-dimensional models of RNA, in preparation

[42] J.M. Wang, P. Cieplak, P.A. Kollman, How well does a restrained electrostatic potential (RESP) model perform in calculating conformational energies of organic and biological molecules, J. Comp. Chem. 21 (2000) 1049-1074. 
[43] D.A. Case, D.A. Pearlman, J.W. Caldwell, T.E. Cheatham III, J. Wang, W.S. Ross, C.L. Simmerling, T.A. Darden, K.M. Merz, R.V. Stanton, A.L. Cheng, J.J. Vincent, M. Crowley, V. Tsui, H. Gohlke, R.J. Radmer, Y. Duan, J. Pitera, I. Massova, G.L. Seibel, U. Singh, P.K. Weiner, P.A. Kollman, AMBER 7, University of California, San Francisco, 2002.

[44] D.A. Case, T.A. Darder, T.E. Cheatham III, C.L. Simmerling, J. Wang, R.E. Duke, R. Luo, K.M. Merz, B. Wang, D.A. Pearlman, M. Crowley, S. Brozell, V. Tsui, H. Gohlke, J. Mongan, V. Hornak, G. Cui, P. Beroza, C. Schafmeister, J.W. Caldwell, W.S. Ross, P.A. Kollman, AMBER 8, University of California, San Francisco, 2004.

[45] H.J.C. Berendsen, J.P.M. Postma, W.F. van Gunsteren, A. DiNola, J.R. Haak, Molecular dynamics with coupling to an external bath, J. Chem. Phys. 81 (1984) 3684-3690.

[46] U. Essmann, L. Perera, M.L. Berkowitz, T.A. Darden, H. Lee, L.G. Pedersen, A smooth particle mesh ewald method, J. Chem. Phys. 103 (1995) 8577.

[47] T.E. Cheatham III, P.A. Kollman, Molecular dynamics simulations highlighting the structural differences among DNA:DNA, RNA:RNA, and DNA:RNA hybrid duplexes, J. Am. Chem. Soc. 119 (1997) 4805-4825.

[48] R. Lavery, H. Sklenar, The definition of generalised helicoidal parameters and of axis curvature for irregular nucleic acids, J. Biomol. Struct. Dyn. 6 (1988) 63-91.
[49] W. Saenger, Principles of nucleic acid structure, in: C.R. Cantor (Ed.), Springer Advanced Texts in Chemistry, Springer-Verlag, New York, U.S.A., 1984.

[50] Y.G. Yingling, B.A. Shapiro, Dynamic behavior of the telomerase RNA hairpin structure and its relationship to dyskeratosis congenita, J. Mol. Biol. 348 (2005) 27-42.

[51] T. Hermann, E. Westhof, Non-Watson-Crick base pairs in RNA-protein recognition, Chem. Biol. 6 (1999) R335-R343.

[52] J. Lin, H. Ly, A. Hussain, M. Abraham, S. Pearl, Y. Tzfati, T.G. Parslow, E.H. Blackburn, A universal telomerase RNA core structure includes structured motifs required for binding the telomerase reverse transcriptase protein, PNAS 101 (2004) 14713-14718.

[53] C.W. Hilbers, P.J. Michiels, H.A. Heus, New developments in structure determination of pseudoknots, Biopolymers 48 (1998) 137-153.

[54] Y. Wang, N.M. Wills, Z. Du, A. Rangan, J.F. Atkins, R.F. Gesteland, D.W. Hoffman, Comparative studies of frame shifting and nonframeshifting RNA pseudoknots: a mutational and NMR investigation of pseudoknots derived from the bacteriophage T2 gene $32 \mathrm{mRNA}$ and the retroviral gagpro frameshift site, RNA 8 (2002) 981-996.

[55] N.B. Leonitis, E. Westhof, Geometric nomenclature and classification of RNA base pairs, RNA 7 (2001) 499-512. 